\section{¿Cómo podemos ayudar a los maestros en formación a analizar los libros de texto de ciencias?}

UTE. Revista de Ciències de l'Educació

Monogràfic 2019. Pàg. 33-42

ISSN 1135-1438. EISSN 2385-4731

http://revistes.publicacionsurv.cat/index.php/ute

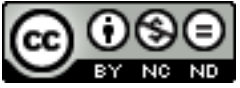

DOI: https://doi.org/10.17345/ute.2019.2

\author{
Francisco Javier Perales Palacios \\ Rebut: 31/05/2019 Acceptat: 18/09/2019
}

\title{
Resumen
}

A pesar de los aires de cambio que vienen soplando sobre la educación obligatoria en España, persisten algunas inercias ajenas a estos cambios, una de ellas es el uso masivo del libro de texto como referente del saber científico en los centros educativos. Ante esta evidencia, es responsabilidad de los formadores de maestros el dotarles de estrategias de análisis de dicho recurso educativo. En este artículo se muestra la forma de trabajar dicha competencia en la Facultad de Ciencias de Educación de la Universidad de Granada, lo que se traduce en un protocolo de análisis que se plantea para ser abordado dentro de la asignatura de Didáctica de las Ciencias para Educación Primaria. Se describen los puntos que incluye dicho protocolo y se cuantifican las dificultades que una muestra de estudiantes presenta en su resolución. Se concluye destacando la oportunidad que representa esta tarea para servir de conexión con el periodo de prácticas escolares y el futuro profesional de los maestros en formación.

Palabras claves: Formación de maestros, libros de texto, ilustraciones, Educación Primaria.

\begin{abstract}
In spite of the winds of change that are blowing over compulsory education in Spain, some inertias that are alien to these changes persist, one of them being the massive use of the textbook as a reference point for scientific knowledge in educational centers. Given this evidence, it is the responsibility of teacher trainers to equip them with strategies for analyzing such educational resource. This article shows how this competence is working in the Faculty of Education Sciences of the University of Granada, which consists of a protocol of analysis as an activity within the subject of Science Education for Education Primary. The points included in this protocol are described so as the difficulties that a sample of students had in their resolution. Finally, the opportunity that this task represents to serve as a connection with the period of school practices and the professional future of the preservice teachers is highlighted.
\end{abstract}

Keywords: Training teachers, textbooks, illustrations, Primary Education. 


\section{Introducción}

Como es bien sabido, atravesamos una época controvertida en cuanto al papel de la escuela en el mundo actual y los métodos de enseñanza que debiera usar para tratar de sintonizar con los profundos cambios sociales, tecnológicos o laborales a los que asistimos. A pesar de los intentos de renovación que alimentan muchas iniciativas que se vienen produciendo, más a nivel teórico que aplicado, en escasas ocasiones se prescinde de un recurso educativo que permanece en los niveles obligatorios de la enseñanza desde hace bastantes décadas. Naturalmente, nos estamos refiriendo al libro de texto. En contadas ocasiones los centros de enseñanza se atreven a prescindir de él y sustituirlo por mecanismos de obtención de información más flexibles y abiertos. Las razones de ello son diversas, aunque podríamos mencionar el sostén que representan para el profesorado y alumnado, las subvenciones que en algunos casos reciben las familias para su adquisición, los intereses económicos de las editoriales e incluso razones de naturaleza ideológica (Perales, 2006a).

Ante esta realidad, los formadores de maestros no podemos eludir la responsabilidad de ofrecer dentro del currículo de los grados universitarios de Primaria la oportunidad de que los estudiantes adquieran mecanismos de análisis de dicho recurso educativo, lo que les ayudaría a la toma de decisiones en su profesión futura.

En este trabajo, de naturaleza descriptiva e innovadora, nos hemos planteado como objetivo general mostrar la forma en que se trabaja este contenido curricular en la asignatura de Didáctica de las Ciencias para Educación Primaria (I. Ciencias del espacio y de la Tierra) en la Facultad de Ciencias de la Educación de la Universidad de Granada, así como explicitar algunas de las dificultades que una muestra de estudiantes de dicha materia halla en su implementación.

\section{Precedentes}

La frecuencia de utilización del libro de texto en la mayoría de las aulas, no solo en nuestro país sino también a nivel internacional, ha generado una fecunda línea de investigación en el ámbito de las ciencias experimentales. De hecho, en la revisión bibliométrica realizada por Aguilera (2019) en la Web of Science durante los últimos ocho años, dicha línea aparece representada en cuarto lugar por el número de publicaciones.

Un material complejo como el libro de texto admite por tanto un análisis multifactorial que en nuestro caso representamos en la Figura 1 y que nos sirvió de base para el diseño del protocolo de análisis objeto de este trabajo.

Dentro de esta variedad de perspectivas de análisis hemos investigado en algunas de ellas: ilustraciones, conexión de estas con el texto, secuencia didáctica, interdisciplinariedad o actividades prácticas. Para ello hemos contado con la perspectiva de los propios investigadores, pero también con la de estudiantes y profesores.

En este último colectivo llevamos a cabo un estudio mediante la técnica de grupo de discusión con una muestra de maestros y profesores de secundaria acerca de la valoración que hacían de los libros de texto en general y de las ilustraciones en particular (Aguilera \& Perales, 2018). Como síntesis del mismo, los docentes participantes opinaban que los libros de texto incidían negativamente en la actitud de los alumnos hacia la ciencia, principalmente debido al uso abusivo que se hace de ellos; en cambio, las ilustraciones sí parecían mejorar el interés de los alumnos por la ciencia y su aprendizaje, así como aportaban un potencial explicativo de los contenidos, aunque dependiendo del uso que de ellas se hiciera en el aula. 


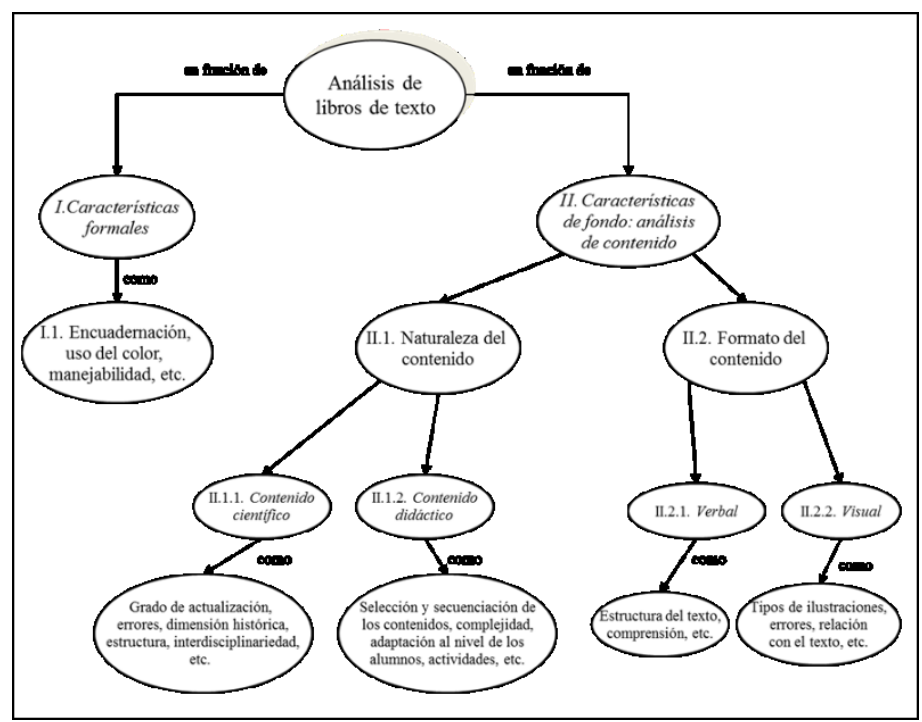

Figura 1. Propuesta de categorías de análisis de libros de texto. Fuente: Perales (2006)a.

De forma complementaria llevamos a cabo un sondeo entre estudiantes y profesores de secundaria sobre el uso habitual que hacían de las ilustraciones (Perales \& Vílchez, 2015). Según los segundos, la importancia de la mayoría de las ilustraciones como recurso de enseñanza es clave; sin embargo, los estudiantes no suelen hacer un uso autónomo de aquellas como herramienta de aprendizaje, a no ser que se les indique expresamente. Estos últimos también valoraban como menos útiles las fotografías y hubo algunas disonancias cuando contrastaban la información contenida en las ilustraciones con la del texto escrito acompañante.

Centrándonos ya de forma más concreta en el propósito de este trabajo, elaboramos una plantilla de evaluación que fue depurada a partir de su aplicación con estudiantes de Pedagogía y de Magisterio de Educación Primaria durante varios cursos académicos, publicando una primera versión (Perales \& Vílchez, 2012) que posteriormente experimentó nuevos ajustes. Dicha plantilla satisface los requisitos que otros estudios internacionales han considerado para un análisis similar (Khine, 2013), incluidos los que hacen referencia a la vertiente pictórica de los libros de texto (Perales \& Jiménez, 2002; Slough, McTigue, Kim \& Jennings, 2010).

En cuanto a los objetivos de aprendizaje que nos planteamos se centran en evaluar la idoneidad de un protocolo como el descrito aquí para la adquisición de la competencia del análisis de libros de texto por parte de los futuros maestros.

En el próximo apartado expondremos el procedimiento seguido en esta actividad con los maestros en formación.

\section{Método}

\subsection{El diseño del currículo}

La asignatura de Didáctica de las Ciencias para Educación Primaria posee una carga lectiva de nueve créditos y se imparte en el primer semestre del tercer curso del grado de Primaria, en un horario de tarde para compatibilizarla con el Practicum I que se lleva a cabo en los colegios en horario de mañana. Combina clases de gran grupo (tres horas semanales) con dos seminarios de $1,5 \mathrm{~h}$ para cada semigrupo. Para las primeras se sigue un manual (Vílchez, Benarroch, Carrillo, Cervantes, Fernández \& Perales, 2018) en el que se plantean actividades individuales para realizar antes de las clases, junto con otras de 
pequeño grupo a cumplimentar en el aula, y contenidos científico-didácticos orientados al currículo de Educación Primaria. Los seminarios son dedicados al análisis de dicho currículo, al de libros de texto (objeto de este trabajo), a prácticas de laboratorio y a la explicación y seguimiento de una unidad didáctica realizada en pequeños grupos.

\subsection{El análisis de libros de texto}

A esta actividad se le dedican dos sesiones semanales de seminario, explicándoles cómo llevarla a cabo y supervisando su ejecución en grupo, dejando una semana más para su envío a través de la plataforma docente de la universidad. Asimismo, en el propio manual de la asignatura disponen de la rejilla de análisis (Figura 2) y unas instrucciones para su desarrollo. Cada grupo utiliza para su trabajo un libro de una editorial elegida libremente, disponiendo en la biblioteca de la facultad de un suficiente número de ejemplares. Posteriormente a la entrega del trabajo al profesor, éste se lo devuelve a cada grupo con las correcciones efectuadas sobre el propio texto que ellos enviaban a través de la plataforma.

\subsection{Descripción de los pasos del análisis (Figura 2)}

\section{Datos completos del libro analizado.}

1. Relación entre contenidos, temas y bloques de temas.

2. Estructura de los temas (por ejemplo, introducción, preguntas iniciales, etc.).

3. Uso de la imagen. Tipos (dibujos, fotografías, tablas...). Cuantificarlas y valorarlas de acuerdo al aldecálogo repartido.

4. Lenguaje utilizado (nivel de comprensión, uso de analogías, sexismo, interculturalidad, glosario...).

5. Contenidos:

5.1. Tipos conceptuales, procedimentales y actitudinales, concretándolos para una unidad

5.2. Adecuación a los objetivos y contenidos de la legislación (de ámbito científico).

5.3. Conexión con el medio.

5.4. Relaciones interdisciplinares: áreas transversales (por ejemplo, Educación Ambiental, Educación para la Salud, etc.) y Ciencia-Tecnología-Sociedad.

5.5. Visión que ofrece sobre la Naturaleza e Historia de la Ciencia.

5.6. Grado de actualización científica.

5.7. Errores conceptuales.

\section{Actividades:}

6.1. Adecuación a los objetivos marcados por la legislación (de ámbito científico) y al desarrollo de las competencias.

6.2. Tipos de actividades.

6.3. Grado de directividad (según sean las instrucciones para su resolución).

6.4. Grado de complejidad.

6.5. Recursos didácticos (materiales que deben utilizarse en las actividades)

6.6. Adecuación al contexto del alumno.

7. Valoración general del libro y propuestas de mejora.

Figura 2. Protocolo utilizado para el análisis de libros de texto. Fuente: adaptado de Perales y Vílchez (2012).

0. Datos. Aquí deben incluirse los datos del libro analizado según las normas APA.

1. Relación. Este punto se refiere a la búsqueda en el libro analizado de referencias explícitas a los contenidos de otros temas (en la introducción al libro, en la forma en que se estructura el índice, en el desarrollo de los distintos temas...), de manera que el alumno pueda percibir que existe relación entre ellos y no aparezcan (como suele suceder) como temas aislados unos de otros y que se van abordando sucesivamente sin saber el porqué. El indicador de esta relación es básicamente si los autores del libro hacen alusión al contenido de otros temas y/o justifican por qué el contenido está organizado de la forma en que se hace (por ejemplo, frases del tipo «Una vez que en el tema anterior mencionamos las fuentes de energía, ahora vamos a estudiar...»). Ello exigirá, por tanto, hacer una lectura general del libro, especialmente el índice general, la introducción y el inicio de cada tema (si estuviera accesible el libro del profesor, sería conveniente comprobar si en él sí se ofrece una justificación de las relaciones buscadas). 
2. Estructura. Extraer la estructura común de los temas del libro, que suele respetarse a lo largo del mismo. Se deben identificar las secuencias de desarrollo de cada uno de ellos (por ejemplo, preguntas iniciales, exposición del contenido, etc.). Ello, a su vez, permitirá ubicar la metodología de enseñanza que subyace en el libro. Para lograrlo los estudiantes ya han visto en las clases de gran grupo cómo categorizar y secuenciar el discurso del libro para identificar su orientación didáctica (transmisiva, constructivista...).

3. Uso de la imagen. Con el fin de que se tome conciencia de la importancia que tienen las ilustraciones en los libros de texto actuales (al menos en cuanto a la superficie que ocupan), cada grupo seleccionará un tema del libro de texto y medirá, con ayuda de una regla, la superficie ocupada por las ilustraciones y la ocupada por el texto escrito, calculando los porcentajes de cada formato para poder compararlos. A continuación, se tratará de clasificar las ilustraciones presentes en el libro. Para ello en el tema del libro elegido en el punto 2 anterior deben seleccionar al azar entre 10 y 15 ilustraciones del mismo. Los criterios que pueden servir para hacerlo se muestran en la Tabla 1 (Jiménez \& Perales, 2001; Perales \& Jiménez, 2002). Tal clasificación se puede llevar a cabo mediante la elaboración de una ficha individual para cada ilustración (incluyéndola escaneada) en la que aparezca cada criterio de la Tabla 1 y cómo se clasifica dicha ilustración según él. Dentro de la misma ficha se puede incorporar la valoración didáctica de la ilustración de acuerdo al decálogo que se muestra en el Figura 3 (con un sí o un no), justificando la relación de los puntos del primero con la ilustración analizada.

4. Lenguaje. Se trata de adquirir una primera aproximación al lenguaje utilizado en el libro. Para ello se debe abordar la lectura de un apartado de un tema, anotando (justificadamente y señalando las frases textuales) los siguientes aspectos:

- Frases o párrafos que presenten dificultad de comprensión (para los estudiantes de esta asignatura y, supuestamente, para los de Educación Primaria).

- Recursos lingüísticos para fomentar la comprensión, como las analogías.

- Referencias que puedan indicar (implícita o explícitamente) algún tipo de discriminación en cuanto a sexo, cultura, religión...

- Si el libro dispone de algún glosario que defina o clarifique los términos más novedosos.

5. Contenidos. Continuando con el análisis de los contenidos del libro de texto, se pide revisarlos de acuerdo a los epígrafes del Figura 2.

Tabla 1: Criterios de clasificación de las ilustraciones de un libro de texto

Criterio de clasificación Descripción del mismo

1. Función didáctica en la que aparecen las ilustraciones

2. Iconicidad.

3. Relación con el texto principal.

4. Etiquetas verbales.
Evocación, definición, aplicación, descripción, interpretación y problematización.

¿Qué semejanza posee la ilustración con la realidad (realistas, realistas con elementos simbólicos, esquemas, gráficos...)?

¿Cómo se relaciona el texto escrito con la ilustración (referencia detallada, breve o no se refiere a ella)?

¿Incorporan textos dentro de la ilustración y/o a pie de la ilustración? 


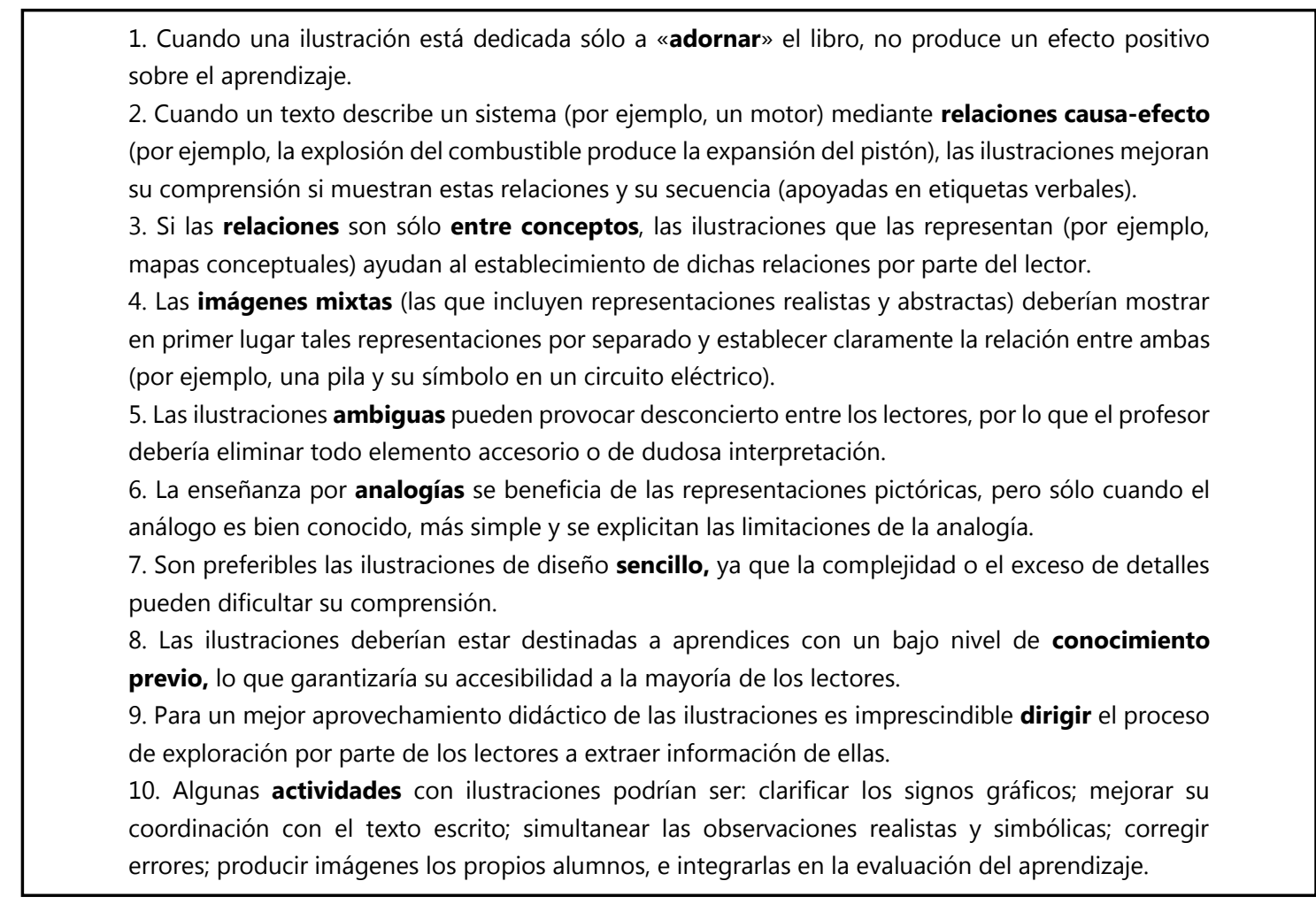

Figura 3. Decálogo para el análisis didáctico de las ilustraciones de un libro de texto

6. Actividades. En este apartado se analizan las actividades de un tema del libro de texto escogido, poniendo los ejemplos oportunos, de acuerdo con los puntos del Figura 2.

7. Valoración. Por último, en este apartado se pretende establecer unas conclusiones generales teniendo en cuenta los puntos fuertes y débiles hallados en el análisis previo, señalando cómo podría intentarse paliar las deficiencias detectadas en el libro.

\subsection{Muestra participante}

Participaron en esta actividad un total de 73 alumnos distribuidos en 15 grupos durante el curso académico 2018-19.

\section{Análisis de Resultados}

La implementación de la actividad llevada a cabo la evaluamos a través de las limitaciones detectadas en los distintos apartados representados en el protocolo del Figura 2. Ello nos proporciona una foto fija de las posibilidades de la propuesta de trabajo descrita, así como de los puntos débiles de la misma, en los que habría que ahondar a través de puestas en práctica futuras.

En la Tabla 2 mostramos el recuento de las dificultades que los grupos de alumnos evidenciaron en sus trabajos. Dicho recuento se ha organizado en función de los epígrafes del Figura 2, mostrando las frecuencias absolutas y los porcentajes sobre el total de las dificultades identificadas. 
Tabla 2: Relación de dificultades evidenciadas en la actividad de análisis de libros de texto por parte de los diferentes grupos de estudiantes $(N=15)$.

\begin{tabular}{|c|c|c|c|}
\hline Apartado Figura2 & Dificultades & Frecuencia & Porcentaje \\
\hline $\begin{array}{l}\text { 1. Relación entre } \\
\text { contenidos, temas y } \\
\text { bloques de temas }\end{array}$ & $\begin{array}{l}\text { - Confunden su apreciación personal con la que } \\
\text { debiera aparecer en el libro } \\
\text { - No la abordan }\end{array}$ & $\begin{array}{l}3 \\
1\end{array}$ & $7 \%$ \\
\hline \multirow[t]{2}{*}{$\begin{array}{l}\text { 2. Estructura de los } \\
\text { temas }\end{array}$} & $\begin{array}{l}\text { - No identifican la metodología de enseñanza } \\
\text { subyacente al libro }\end{array}$ & 6 & \multirow[t]{2}{*}{$12 \%$} \\
\hline & - No concretan suficientemente la estructura & 1 & \\
\hline 3. Uso de la imagen & $\begin{array}{l}\text { - Clasifican erróneamente las ilustraciones según su } \\
\text { iconicidad } \\
\text { - No calculan el porcentaje parcial o global de } \\
\text { superficie ocupada por el texto escrito y por las } \\
\text { ilustraciones } \\
\text { - No hacen la valoración de las ilustraciones de } \\
\text { acuerdo al decálogo } \\
\text { - Atribuyen más de una función didáctica a las } \\
\text { ilustraciones } \\
\text { - No clasifican las ilustraciones de acuerdo a todos los } \\
\text { criterios de la Tabla } 1 \\
\text { - No justifican las clasificaciones } \\
\text { - Clasifican incorrectamente las ilustraciones según su } \\
\text { función didáctica } \\
\text { - No concretan las medidas de superficie realizadas }\end{array}$ & $\begin{array}{l}4 \\
4 \\
3 \\
2 \\
1 \\
1 \\
1 \\
1\end{array}$ & $29 \%$ \\
\hline 4. Lenguaje utilizado & & 0 & $0 \%$ \\
\hline \multirow[t]{5}{*}{ 5. Contenidos } & $\begin{array}{l}\text { - No cuantifican las tipologías de contenidos (5.1) } \\
\text { - Confunden tipologías de contenidos }\end{array}$ & $\begin{array}{l}3 \\
1\end{array}$ & \multirow[t]{5}{*}{$34 \%$} \\
\hline & $\begin{array}{l}\text { - No justifican la adecuación de los objetivos y } \\
\text { contenidos a la legislación (5.2) }\end{array}$ & 7 & \\
\hline & $\begin{array}{l}\text { - No consideran como áreas transversales la Educación } \\
\text { Ambiental y la Educación para la Salud (5.4) } \\
\text { - No identifican contenidos Ciencia-Tecnología- } \\
\text { Sociedad } \\
\text { - No ejemplifican los contenidos } \\
\text { - No ejemplifican las áreas transversales }\end{array}$ & $\begin{array}{l}3 \\
1 \\
1 \\
1\end{array}$ & \\
\hline & - No reconocen la naturaleza de la ciencia (5.5) & 2 & \\
\hline & - No se abordan los puntos 5.3-5.7 & 1 & \\
\hline \multirow[t]{3}{*}{ 6. Actividades } & $\begin{array}{l}\text { - No justifican la adecuación a los objetivos marcados } \\
\text { por la legislación y al desarrollo de las competencias } \\
\text { (6.1) }\end{array}$ & 5 & \multirow[t]{3}{*}{$15 \%$} \\
\hline & $\begin{array}{l}\text { - No ejemplifican los tipos de actividades (6.2) } \\
\text { - No comprenden un tipo de actividad }\end{array}$ & $\begin{array}{l}1 \\
1\end{array}$ & \\
\hline & - No ejemplifican el grado de directividad (6.3) & 2 & \\
\hline $\begin{array}{l}\text { 7. Valoración general } \\
\text { del libro }\end{array}$ & & 0 & $0 \%$ \\
\hline Otras deficiencias & $\begin{array}{l}\text { - Analizan un tema de Ciencias Sociales } \\
\text { - Equivocan el apartado del Figura } 2\end{array}$ & $\begin{array}{l}1 \\
1\end{array}$ & $3 \%$ \\
\hline Total & & 59 & $100 \%$ \\
\hline
\end{tabular}


Si nos detenemos en analizar las deficiencias halladas, en primer lugar podemos observar que la frecuencia media por trabajo de grupo es de 3,9 (número total de dificultades dividido por el número de grupos), aunque hay una considerable desviación típica en las mismas.

Globalmente el apartado que acumula más dificultades es el referido a los contenidos del libro de texto, seguido de cerca del referido a las ilustraciones. Hemos de tener en cuenta que el primero es el que requería más ítems que cumplimentar aunque comparativamente las ilustraciones superan claramente en dificultades al de las actividades.

Refiriéndonos más específicamente a cada uno de los apartados del Figura 2, podemos destacar lo siguiente:

Apartado 1. Relación entre contenidos, temas y bloques de temas. A algunos grupos les cuesta diferenciar entre la coherencia que para ellos aparentemente suele presentar el índice del libro frente a cómo realmente se explicita en el mismo. Esta es una deficiencia muy común entre los libros de texto manejados, es decir, existen saltos de unos temas a otros sin solución de continuidad. A no ser que los maestros suplan esta carencia, a los alumnos les resulta difícil interpretar qué hilo conductor preside el discurrir de los temas.

Apartado 2. Estructura de los temas. A pesar de haber trabajado en el gran grupo los modelos didácticos más comunes en la enseñanza de las ciencias, la "traducción" de la secuencia del texto que incluyen los libros en modelos reconocibles supone una dificultad presente en un número significativo de los trabajos analizados. Sin duda, la modelización es una operación cognitiva compleja, incluso para los maestros en formación.

Apartado 3. Uso de la imagen Esta actividad que se propone a los grupos de estudiantes representa una novedad para la mayoría, aunque, como hemos indicado en los precedentes de este trabajo, es absolutamente necesario que los futuros maestros se alfabeticen visualmente (Perales, 2006b). Ello debiera pasar, a nuestro juicio, porque sepan diferenciar las ilustraciones según determinados criterios y valorarlas didácticamente. Aunque, no muy significativas, se presentan dificultades en las tareas indicadas; no obstante, también llama la atención que en algunos casos no sepan calcular las superficies relativas ocupadas por el texto escrito y las ilustraciones.

Apartado 5. Contenidos. La dificultad más extendida tiene que ver con la ausencia de justificación en la adecuación de los objetivos y contenidos a la legislación vigente en el momento de edición del libro de texto analizado. Ello requiere de la identificación de la ley de educación correspondiente y la comparación de los objetivos y contenidos prescritos para las ciencias de Primaria con los incluidos en el libro de texto; más que una tarea compleja cognitivamente supone un trabajo de análisis documental que no todos los grupos llevaron a cabo, pero necesaria si se quiere evaluar la adecuación de la editorial seleccionada

Apartado 6. Actividades. Al igual que ocurría en el apartado anterior, la deficiencia más frecuente está relacionada con la valoración de la adecuación a los objetivos marcados por la legislación y al desarrollo de las competencias, y la posible explicación de la misma coincide con la apuntada en dicho apartado.

\section{Conclusiones}

A través de este artículo hemos pretendido mostrar cómo puede trabajarse con los maestros de Primaria en formación una actividad que intenta dotarles de la competencia de analizar y evaluar los libros de texto de ciencias, tarea que tarde o temprano deberán afrontar cuando se conviertan en maestros en 
ejercicio, así como las dificultades que encuentran en su implementación. Esto es especialmente deseable en lo que corresponde a las ilustraciones, dado que ocupan un espacio muy relevante en los libros actuales. Los condicionantes que guían la elección de una editorial determinada por parte del equipo docente de una escuela son diversos, pero un maestro debe de disponer de un espíritu crítico tanto si la elección le viene dada como si posee algún margen de maniobra al respecto.

Vistos los resultados sintéticamente parece que, en general, los grupos de estudiantes son capaces de afrontar el análisis de libros de texto con cierta solvencia. No obstante, existen algunas actividades parciales que les suponen en mayor o menor medida algún grado de dificultad. En concreto:

- Traducir en modelos didácticos la secuencia de enseñanza que siguen los diferentes libros.

- Clasificar y valorar las ilustraciones de los libros de acuerdo a criterios previos.

- Contrastar los contenidos y actividades del libro con los objetivos, contenidos y competencias prescritos por la legislación educativa vigente en la fecha de edición.

- Reconocer los elementos transversales del currículo (p. ej., Educación Ambiental y Educación para la Salud) en el desarrollo de los temas del libro.

Por otro lado, los estudiantes participantes en este estudio no evidenciaron dificultades significativas en el análisis del lenguaje del libro y fueron capaces de realizar una valoración final del mismo. Quedaría pendiente para el futuro contrastar los resultados obtenidos con otros estudios internacionales y los criterios de deseabilidad didáctica para poder extraer conclusiones respecto a la idoneidad de los libros objeto de análisis.

La actividad descrita constituye además una oportunidad excelente para la toma de contacto con el principal recurso didáctico que se hallarán cuando desarrollen su periodo de prácticas en la escuelas, así como de los desafíos que para ellos supone la transposición didáctica del contenido científico a la edad y nivel educativo de sus futuros alumnos.

\section{Referencias bibliográficas}

Aguilera, D. (2019). Actitud hacia la ciencia y los factores moderadores: metodología participativa, ilustraciones, libros de texto y estilos de aprendizaje. Tesis Doctoral. Universidad de Granada.

Aguilera, D. \& Perales, F.J. (2018). El libro de texto, las ilustraciones y la actitud hacia la Ciencia del alumnado: percepciones, experiencias y opiniones del profesorado. Enseñanza de las Ciencias, 36(3), 4158.

Jiménez, J. D. \& Perales, F. J. (2001). El análisis secuencial del contenido. Su aplicación al estudio de libros de texto de Física y Química. Enseñanza de las Ciencias, 19(1), 3-19.

Khine, M. S. (Ed.) (2013). Critical Analysis of Science Textbooks. Evaluating instructional effectiveness. Springer: Perth, Australia.

Perales, F. J. \& Jiménez, J. D. (2002). Las ilustraciones en la enseñanza-aprendizaje de las ciencias. Análisis de libros de texto. Enseñanza de las Ciencias, 20(3), 369-386. 
Perales, F.J. (2006a). Pasado, presente y ¿futuro? de los libros de texto. Alambique, 48, 57-63.

Perales, F. J. (2006b). Uso (y abuso) de la imagen en la enseñanza de las ciencias. Enseñanza de las Ciencias, 24(1), 13-30

Perales, F. J. \& Vílchez, J.M. (2012). Libros de texto: ni contigo ni sin ti tienen mis males remedio. Alambique, 70, 75-82.

Perales, F. J. \& Vílchez, J. M. (2015). Iniciación a la investigación educativa con estudiantes de secundaria: el papel de las ilustraciones en los libros de texto de ciencias. Enseñanza de las Ciencias, 33(1), 243-262.

Slough, S., McTigue, E., Kim, S. \& Jennings, S. K. (2010). Science textbooks' use of graphical representation: a descriptive analysis of four sixth grade science texts. Reading Psychology, 31,301-325.

Vílchez, J.M. (coord.), Benarroch, A., Carrillo, F.J., Cervantes, A., Fernández, M. \& Perales, F.J. (2018, $3^{\mathrm{a}}$ ed.). Didáctica de las Ciencias para Educación Primaria (I. Ciencias del espacio y de la Tierra). Madrid: Pirámide. 